\title{
Point-of-care hand-held ultrasound for peripheral intravenous cannulation: a scoping review protocol
}

\author{
*Priscilla Pather, ${ }^{1,2,3}$ Jessica A. Schults, ${ }^{1,2,4}$ Claire M. Rickard, ${ }^{1,2,5,6,7}$ and Gillian Ray-Barruel ${ }^{1,2,3,5,6}$ \\ 1 Alliance for Vascular Access Teaching and Research Group, Menzies Health Institute Queensland, Griffith University, QLD, Australia \\ 2 School of Nursing and Midwifery, Griffith University, Nathan, QLD, Australia \\ ${ }^{3}$ QEll Jubilee Hospital, Coopers Plains, QLD, Australia \\ ${ }^{4}$ Department of Anaesthesia and Pain Management, Queensland Children's Hospital, QLD, Australia \\ ${ }^{5}$ Nursing and Midwifery Research Centre, Royal Brisbane and Women's Hospital, QLD, Australia \\ ${ }^{6}$ Nursing Practice Development Unit, Princess Alexandra Hospital, Woolloongabba, QLD, Australia \\ 7 School of Nursing, Midwifery and Social Work, the University of Queensland, QLD, Australia \\ *Corresponding author: Priscilla.Pather@health.qld.gov.au
}

Keywords point-of-care ultrasound, hand-held/handheld ultrasound, peripheral intravenous catheter/cannula, intravenous cannula For referencing Pather $P$ et al. Point-of-care hand-held ultrasound for peripheral intravenous cannulation: a scoping review protocol . Vascular Access 2021; 7(1):16-21.

DOI https://doi.org/10.33235/va.7.1.16-21

\begin{abstract}
Background Billions of peripheral intravenous catheters (PIVCs) are used globally each year, with 44-58\% of first attempts failing. Ultrasound use for PIVC insertion has demonstrated improved first pass success, reduced attempts, and improved satisfaction. With the expansion of point-of-care and hand-held ultrasound units, there is a need to identify and explore options for clinical use.

Aim We aim to conduct a scoping review of peer-reviewed and non-peer-reviewed literature to identify articles that explore the nature and distribution of research activity and current state of evidence in the adoption and use of point-of-care and hand-held ultrasound for PIVC insertion.

Methods A search using keywords and Medical Subject Headings for published and unpublished literature in English between the years 2000 to 2021 will be undertaken in electronic databases and clinical trial registry sites. Two independent reviewers will screen all titles and abstracts for eligibility and extract data from relevant articles into a standardised electronic data collection form. Quantitative studies will be classified into groups investigating similar interventions, strategies and outcomes, and data presented as descriptive statistics, as appropriate. A thematic analysis will be conducted on the information extracted from qualitative studies and findings presented using narrative synthesis.
\end{abstract}

Ethics and dissemination Ethics approval is not required for this review. The findings will be published and presented to audiences invested in peripheral intravenous cannulation success.

\section{Background}

Billions of peripheral intravenous catheters (PIVCs) are used globally each year, ${ }^{1}$ with the United States alone reporting annual sales of over 330 million. ${ }^{2}$ Up to $80 \%$ of hospital inpatients require a short PIVC inserted ${ }^{3,4}$ to deliver intravenous (IV) medications, fluids, blood products and nutritional supplements. ${ }^{2}$ In Queensland, Australia, an estimated 2.75 million vascular access devices were used in public hospitals in 2016, at a total cost of A\$59.14 million - A\$48.85 million for labour and $A \$ 10.17$ million for equipment. 5

Most clinicians overwhelmingly favour PIVCs as the device of choice for delivery of IV treatments. ${ }^{4}$ Traditionally, PIVCs are inserted using landmark, visualisation, and palpation techniques to locate target vessels. ${ }^{6}$ These methods can be difficult, and success depends largely on highly skilled clinicians with considerable cannulation experience.? It has been suggested that knowledge in the assessment and selection of vessels for venous puncture is largely lacking, ${ }^{8}$ making it time-consuming, with $44-58 \%$ first attempts failing and clinicians resorting to 'blind' techniques based on trial and error. ${ }^{9}$

Difficult intravenous access (DIVA) in some patients is even more challenging, particularly for individuals with extreme values in body mass index (obesity or cachexia), oedema, chronic diseases, multiple comorbidities, vasculopathy, history of repeated IV 
injections or IV drug use, chemotherapy, dehydration, and extremes of age..$^{10}$ In such patients, suitable vessels cannot be visualised nor palpated, resulting in clinicians abandoning and escalating to more senior staff or vascular access specialists, if available. ${ }^{11}$ It is estimated that DIVA affects $10-24 \%$ of adults and $37 \%$ of children requiring a PIVC ${ }^{10}$; consequently, some patients have experienced up to 20 needle puncture attempts. ${ }^{12-15}$ Notably, DIVA is exacerbated by clinician limitations such as lack of knowledge and experience of vascular anatomy, suitable vessel location, vessel assessment and selection skills, lack of awareness, information and experience using vein location and visualisation aids and devices. ${ }^{16}$ These factors ultimately cause delays in PIVC insertion.

Delays in successful PIVC insertion result in delayed treatment, asserting the claim that "treatment delayed is treatment denied" in all arenas of healthcare, as the relationship between time to treatment and outcomes are clearly demonstrated. ${ }^{17,18}$ These include delays in diagnosis using IV contrast, commencing IV treatments, and missed IV medication, often culminating in the insertion of a central venous access device. ${ }^{19,20}$ Nye et al. ${ }^{11}$ demonstrated that a treatment delay of up to more than 5 hours can result from the time medication is prescribed to PIVC insertion in DIVA patients. Consequently, clinicians faced with DIVA adopt a mindset of "just get a cannula in any vein",16 thus, often inserting PIVCs into smaller, superficial veins with low blood flow, or other suboptimal anatomical sites (eg, hands and ante-cubital fossa') after multiple failed attempts, resulting in early PIVC failure. ${ }^{21}$ This has prompted the development of a clinical pathway using ultrasound for managing DIVA to improve overall cannulation success, first attempt pass success, vessel health preservation ${ }^{22}$ and reducing pain and trauma. ${ }^{19}$

Ultrasound technology use in healthcare has made viewing and interpreting of anatomical structures as images in real-time highly valuable and its use in intensive care for central and arterial line placement is well documented. ${ }^{23,24}$ The technology uses vibrations (or sound waves) produced by tiny piezoelectric crystals within the transducer (probe). Ultrasonic waves are directed into a body part, hit tissues and structures, and echoes are reflected back to the transducer. These reflected waves are converted into images that display fluid, blood, soft tissue, or bone in tones of grey and black on a screen..$^{25}$

Teachings for PIVC insertion recommend that selected vessel and insertion site must be free from thrombi or atheromatous plaques, superficial, away from valves, have a straight, predictable pathway, have an anteroposterior diameter $\geq 4 \mathrm{~mm}$, skin to vessel distance $\leq 16 \mathrm{~mm}$, and accessed in a manner that avoids damage to key adjacent structures.6,27 It stands to reason that adherence to these principles may be difficult (almost impossible) using conventional landmark, visualisation, or palpation techniques, as the clinician remains blinded to vessel health and anatomy without real-time ultrasound visualisation.
Ultrasound use for PIVC insertion has demonstrated improved first-pass success, reduction in the number of attempts, improved patient satisfaction, and reduced overall procedurerelated complications. ${ }^{27}$ In fact, ultrasound has become the equipment of choice for accessing and securing vascular and arterial access in intensive care patients with decreased reliance on conventional techniques. ${ }^{28,29}$

Despite the commercial availability of ultrasound units, the most frequently cited reason and barrier to a wider uptake and adoption into clinical practice has been limited access to equipment, ${ }^{8}$ with researchers suggesting a need for more units in clinical areas with high PIVC insertion activity. ${ }^{11,30}$ One study suggested factoring transport time of units as part of the procedure to calculate time to successful cannulation, ${ }^{9}$ as a significant amount of time is employed to source and haul ultrasound equipment to point-of-care patient settings.

The advancement of ultrasound technology has led to the development of more compact units at lower cost (https:// www.medicalexpo.com/medical-manufacturer/ultrasoundsystem-17689.html). So called point-of-care ultrasound (POCUS) is becoming more widely available in emergency departments, hospital inpatient settings, community and general practice, rural and remote health settings, and in individuals' homes. ${ }^{31}$ With traditional ultrasound carts weighing around $20 \mathrm{~kg}$ and some POCUS devices just $0.4 \mathrm{~kg}$, its use in medical diagnostics and interventions at the bedside across all patient settings is becoming the norm. ${ }^{31}$ The convenience of POCUS has, unsurprisingly, led to an increase in the uptake and use of ultrasound to assist with cannulation in both adult and paediatric patients. ${ }^{31}$ Evidence supporting its application is increasing, particularly in DIVA and paediatric patients. ${ }^{32-34}$

Recently, there has been an emergence of compact ultrasound units and miniaturisation of viewing platforms in the form of tablets, phone screens, and inbuilt probe screens. ${ }^{35}$ Hand-held point-of-care ultrasound (HH-POCUS) and pocket ultrasound devices herald a potential revolution in technology to support care and have extended the clinical value of POCUS to healthcare workers who frequently insert difficult PIVCs. Definitions and specifications for hand-held and pocket ultrasound are not clearly described in literature or clinical settings. The emerging consensus is that HH-POCUS devices have been described as being the size of a palm or able to fit a physician's pocket ${ }^{35,36}$ and weigh around $400 \mathrm{~g} .{ }^{37,38}$

Despite this innovation, little is known about POCUS and $\mathrm{HH}$-POCUS device use in clinical settings. This includes how $\mathrm{HH}$-POCUS devices compare to traditional, larger ultrasound units and other vein-finding devices in relation to cost, features, image quality, reliability, usability, limitations, training and education, cannulation success, infection control, care of equipment, and user experience. ${ }^{39}$ Many clinicians have resorted to ad hoc 
training and education on ultrasound use for PIVC insertion as expectations for professional development and learning become more self-directed. ${ }^{40}$ Standardised ultrasound training programs and clinical guidelines are lacking, and their development has been overtaken by the implementation and integration of these devices into clinical practice. ${ }^{31,41}$ POCUS was listed as the second health technology hazard for 2020 as safeguards, training, experience, and skill has been outpaced by its adoption. ${ }^{42}$

\section{Methods}

\section{Aim}

The overarching aim of this scoping review is to explore the current state of evidence and gaps in the adoption and use of POCUS and HH-POCUS in clinical settings and the nature and distribution of research activity focusing on POCUS and $\mathrm{HH}$-POCUS for PIVC insertion.

\section{Objectives}

Using Anderson et al's components and elements for scoping studies ${ }^{43}$ these are the objectives of this review:

1. map the scope and range of literature relating to POCUS and $\mathrm{HH}-\mathrm{POCUS}$

2. understand current nomenclature and concepts relating to the definitions and adoption frameworks for POCUS and $\mathrm{HH}-\mathrm{POCUS}$; and,

3. identify gaps in the literature on POCUS and HH-POCUS and ascertain areas for further inquiry in relation to POCUS and $\mathrm{HH}$-POCUS to optimise use and clinical impact in the context of PIVC insertions.

\section{Methodological framework}

This review will be underpinned by the Arksey and O'Malley scoping review framework. ${ }^{44}$ There are five stages of the review: (1) identifying the review questions; (2) identifying relevant literature; (3) charting the data; (4) study selection; and, (5) collating, summarising, and reporting the results.

The Arksey and O'Malley framework was chosen because it enables the examination of the breadth of evidence published on the topic and accommodates a variety of study designs. It is useful for the synthesis of a range of information and evidence and can be used as an overview to map existing literature in the field of POCUS and HH-POCUS in terms of its appropriateness, meaningfulness, and feasibility for clinical practice in the field of PIVC insertion. ${ }^{45}$ Given the new developments around ultrasonography, the review will provide a preliminary survey of the focus, themes, volume, and spread of published literature. ${ }^{43,44,46}$ It will help clarify working definitions and conceptual boundaries ${ }^{45}$ for POCUS and HH-POCUS for PIVC insertion, which have not yet been comprehensively reviewed and explored.

\section{Stage 1: identifying the review questions}

1a. What are the characteristics of studies focusing on POCUS and HH-POCUS for PIVC insertion, conducted since the year 2000 ? (eg, quantitative: randomised controlled trials, quasiexperimental, cohort studies, case control; qualitative: surveys, interviews, focus groups).

1b. What POCUS and HH-POCUS equipment, population, demographics, and clinical settings (hospital, clinic, home environment, etc) feature in the selected literature?

1c. What outcome measures on POCUS and HH-POCUS for PIVC insertion have been reported in the literature? (eg, number of insertion attempts, time to insertion, PIVC outcomes, complications, or failure).

1d. What interventions and education strategies for teaching and learning have been adopted for the promotion, use and support of POCUS and HH-POCUS for PIVC insertion, and what measures define competence and expertise?

1e. What is the experience and/or perspective of clinical users and patients towards POCUS and HH-POCUS for PIVC insertion?

\section{Stage 2: identifying relevant literature}

The review will focus on qualitative and quantitative experimental or quasi-experimental studies that compared POCUS and $\mathrm{HH}$-POCUS devices with either traditional ultrasound equipment or landmark, visualisation, and palpation methods for PIVC insertion. It will identify, appraise, and report outcomes such as cannulation success, time to successful cannulation, number of PIVC attempts, and user experiences with POCUS and HH-POCUS devices, either as user opinion or verdict or in comparison with traditional ultrasound equipment or landmark, visualisation, and palpation methods. This scoping review will include studies on participants of all ages to establish the breadth of evidence and literature.

\section{Search strategy}

The search strategy aims to find both published and unpublished literature. A three-step search strategy will be used, with guidance from a university health librarian. An initial limited search of Medline will be undertaken, followed by analysis of text words contained in the title and abstract, and of the index terms used to describe the article. A second search using all identified keywords, index terms, and MeSH headings will then be undertaken across the following databases: PubMed, Cumulative Index to Nursing and Allied Health (CINAHL), Excerpta Medica Database (Embase), Web of Science, Scopus, Cochrane CENTRAL, Google Scholar, Clinicaltrials.gov, Australian New Zealand Clinical Trials Registry, and International Clinical Trials Registry Platform. The search for unpublished studies will include ProQuest Dissertations and Theses, Open Access Theses and Dissertations and National Grey Literature Collection (MedNar). Finally, the reference lists of all selected articles will be searched for additional studies. Studies 
published in English between the years 2000-2021 will be considered for inclusion in this review. This limitation has been set because ultrasound-guided PIVC insertion was not common prior to 2000.

\section{Initial keywords}

Point-of-care ultrasound, hand-held/handheld ultrasound, POCUS, pocket ultrasound, portable ultrasound, peripheral intravenous catheter/cannula, intravenous cannula, peripheral venous catheter, short peripheral catheter, and PIVC/PVC/PIV/ SPC

\section{Stage 3: charting the data}

Studies will be selected based on phenomena of interest and inclusion and exclusion criteria.

\section{Types of intervention(s)/phenomena of interest}

- Point-of-care ultrasound and hand-held point-of-care ultrasound for PIVC insertion in live participants in all age groups and settings.

\section{Inclusion criteria}

- Qualitative or quantitative, experimental, or quasiexperimental studies comparing HH-POCUS devices with either traditional ultrasound equipment or landmark, visualisation, and palpation methods for PIVC insertion.

- Qualitative studies reporting data on user and patient experiences and satisfaction with HH-POCUS devices, either as a user opinion or verdict or in comparison with traditional ultrasound equipment or landmark, visualisation, and palpation methods for PIVC insertion.

- Studies including participants of all ages using POCUS- and/ or HH-POCUS-guided PIVC insertion.

\section{Exclusion criteria}

- Insertion of central venous access devices including central venous lines and peripherally inserted central catheters using POCUS or HH-POCUS.

- Insertion of arterial lines using POCUS or HH-POCUS.

- Studies using phantom arms or vessels.

- Animal studies.

- Letters to editors, opinions, and editorials commenting on POCUS or HH-POCUS.

Initial selection will use title appraisal for suitability, remaining studies will be appraised by abstract and, finally, remaining studies will undergo full text appraisal. Two reviewers will assess all results to increase robustness of study selection. If any discrepancy arises, a third reviewer will be engaged.

\section{Stage 4: study selection}

Bibliographic software package EndNote X9 (Clarivate) will be used as a reference management tool and Microsoft Excel (2010) will be used to record extracted data and information.

A standardised data extraction form in Excel will be used to extract the following data, if available and applicable:

1. Author(s).

2. Year of publication.

3. Country of origin.

4. Concept/theme.

5. Aims/purpose.

6. Setting (inpatient/outpatient, hospital/clinic/home).

7. Study population (eg, adults, paediatrics, neonates), setting (emergency department, intensive care unit, medical, surgical, oncology, outpatients, etc) and sample size.

8. Methodology/study type.

9. Intervention type and comparator.

10. Duration of the intervention.

11. Who collected the data.

12. Quantitative clinical outcomes (eg, cannulation success; time to successful cannulation, number of attempts, PIVC longevity, PIVC failure).

13. Qualitative outcomes (eg, clinician/user experience and/or satisfaction, patient experience and/or satisfaction, parent of child experience and/or satisfaction).

14. Implementation strategies (eg, education, training, resources).

15. Grant/funding support.

\section{Stage 5: collating, summarising, and reporting the results}

The data will be compiled in an Excel spreadsheet for analysis. All studies will be categorised and presented by tabulating study design, year of publication, country where research was undertaken, and phenomena of interest. Quantitative studies will be classified into groups investigating similar interventions, strategies, and outcomes, and data will be presented in narrative format or statistical tables, as appropriate. Descriptive statistics will be used to categorise the characteristics of the studies, where possible and appropriate. A thematic analysis will be conducted on the information extracted from qualitative studies. Intervention outcomes and outcome measures will be collected but not analysed for their significance or quality as this is a scoping review. Two members of the team will categorise the studies and any discrepancies will be resolved independently by a third reviewer.

The Consolidated Framework for Implementation Research $(\mathrm{CFIR})^{46}$ will be used to evaluate studies on POCUS and $\mathrm{HH}$-POCUS device implementation into clinical settings for PIVC insertion. Interventions that promote dissemination and prolong the sustainability of POCUS and HH-POCUS in 
clinical settings locally, nationally, and internationally will be appraised. This framework comprises five major domains: (1) intervention characteristics; (2) outer setting; (3) inner setting; (4) characteristics of the individuals involved; and, (5) process of implementation. The framework further has each domain focusing on processes and elements necessary for implementing change (Table 1). These domains and elements will be considered when appraising the literature and results will be classified according to processes and elements relevant to POCUS and $\mathrm{HH}$-POCUS implementation in each domain.

Research gaps will be identified by assessing the volume and quality of studies across the CFIR domains and elements. Areas that are either under-explored or have not been explored will be captured. Missing pieces in the research literature relevant to population or sample (size, type, and location), research method, data collection and/or analysis, and other research variables or conditions, will be highlighted as they emerge from scoping and surveying the literature.

\section{About the authors}

Priscilla Pather, RN MAppSc (Research). Jessica A. Schults, RN PhD. Claire M. Rickard, RN PhD. Gillian Ray-Barruel, RN PhD.

\section{Conflict of interest}

Priscilla Pather has received a NHMRC partnership grant - Difficult peripheral intravenous catheter insertion: Australian considerations for sustainable implementation of ultrasound-guided procedures - and a Griffith University Postgraduate Research Scholarship.
Jessica Schults has received grant funding from Griffith University, Children's Hospital Foundation, and investigator-initiated research and educational grants provided to Griffith University by vascular access product manufacturers (Baxter, Becton Dickinson, Entrotech Life Sciences), unrelated to this project. Claire Rickard reports investigator-initiated research grants and speaker fees provided to Griffith University from vascular access product manufacturers (3M; BD-Bard; Cardinal Health), unrelated to this project. Gillian Ray-Barruel reports investigator-initiated research grants, speaker fees, and consultancy payments provided to Griffith University by product manufacturers (3M, Becton Dickinson, Medline) and education providers (Ausmed, Wolters Kluwer), unrelated to this project.

\section{References}

1. Alexandrou E, Ray-Barruel G, Carr PJ, Frost SA, Inwood S, Higgins N, et al. Use of short peripheral intravenous catheters: characteristics, management, and outcomes worldwide. J Hosp Med. 2018 May 30:13(5).

2. Hadaway L. Short peripheral intravenous catheters and infections. J Infus Nurs. 2012;35(4):230-40

3. Tuffaha H, Rickard C, Webster J, Marsh N, Gordon L, Wallis M, et al. Costeffectiveness analysis of clinically-indicated versus routine replacement of peripheral intravenous catheters. Appl Health Econ Health Policy. 2014 Feb;12(1):51-8

4. Carr PJ, Rippey JCR, Cooke M, Bharat C, Murray K, Higgins N, et al. Development of a clinical prediction rule to improve peripheral intravenous cannulae first attempt success in the emergency department and reduce post insertion failure rates: the Vascular Access Decisions in the Emergency Room (VADER) study protocol. BMJ Open. 2016 Feb 11;6(2):e009196.

5. Tuffaha HW, Marsh N, Byrnes J, Gavin N, Webster J, Cooke M, et al. Cost of vascular access devices in public hospitals in Queensland. Aust Health Rev. 2018;43(5):511-5.

\section{Table 1. The Consolidated Framework for Implementation Research ${ }^{46}$}

\begin{tabular}{|c|c|c|c|c|}
\hline $\begin{array}{l}\text { Unadapted } \\
\text { intervention }\end{array}$ & Outer setting & Inner setting & Individuals & Process \\
\hline $\begin{array}{l}\text { Intervention Source } \\
\text { Evidence Strength \& Quality } \\
\text { Relative Advantage } \\
\text { Adaptability } \\
\text { Trialability } \\
\text { Complexity } \\
\text { Design Quality \& Packaging } \\
\text { Cost }\end{array}$ & $\begin{array}{l}\text { Patient Needs \& Resources } \\
\text { Cosmopolitanism } \\
\text { Peer Pressure } \\
\text { External Policy \& Incentives }\end{array}$ & $\begin{array}{l}\text { Structural Characteristics } \\
\text { Networks \& Communications } \\
\text { Culture } \\
\text { Implementation Climate } \\
\text { Tension for Change } \\
\text { Compatibility } \\
\text { Relative Priority } \\
\text { Organizational Incentives \& } \\
\text { Rewards } \\
\text { Goals and Feedback } \\
\text { Learning Climate } \\
\text { Readiness for } \\
\text { Implementation } \\
\text { Leadership Engagement } \\
\text { Available Resources } \\
\text { Access to Knowledge \& } \\
\text { Information }\end{array}$ & $\begin{array}{l}\text { Knowledge \& Beliefs about } \\
\text { the Intervention } \\
\text { Self-efficacy } \\
\text { Individual Stage of Change } \\
\text { Individual Identification with } \\
\text { Organization } \\
\text { Other Personal Attributes }\end{array}$ & $\begin{array}{l}\text { Planning } \\
\text { Engaging } \\
\text { Opinion Leaders } \\
\text { Formally Appointed Internal } \\
\text { Implementation Leaders } \\
\text { Champions } \\
\text { External Change Agents } \\
\text { Executing } \\
\text { Reflecting \& Evaluating }\end{array}$ \\
\hline
\end{tabular}

Reprinted with author's permission. (c) 2009 Laura Damschroder. 
6. Panebianco NL, Fredette JM, Szyld D, Sagalyn EB, Pines JM, Dean AJ. What you see (sonographically) is what you get: vein and patient characteristics associated with successful ultrasound-guided peripheral intravenous placement in patients with difficult access. Acad Emerg Med. 2009;16(12):1298-303.

7. Cooke M, Ullman AJ, Ray-Barruel G, Wallis M, Corley A, Rickard CM. Not "just" an intravenous line: Consumer perspectives on peripheral intravenous cannulation (PIVC). An international cross-sectional survey of 25 countries. PloS One. 2018;13(2):e0193436 doi: 10.1371/journal. pone.0193436.

8. de la Torre-Montero J-C, Montealegre-Sanz M, Faraldo-Cabana A, OlivaPellicer B, García-Real I, Fenwick M, et al. Venous International Assessment, VIA scale, validated classification procedure for the peripheral venous system. J Vasc Access. 2014;15(1):45-50.

9. Aponte H, Acosta S, Rigamonti D, Sylvia B, Austin P, Samolitis T. The use of ultrasound for placement of intravenous catheters. AANA journal. 2007;75(3):212-6.

10. Rodriguez-Calero MA, Fernandez-Fernandez I, Molero-Ballester LJ, Matamalas-Massanet C, Moreno-Mejias L, de Pedro-Gomez JE, et al. Risk factors for difficult peripheral venous cannulation in hospitalised patients. Protocol for a multicentre case-control study in 48 units of eight public hospitals in Spain. BMJ Open. 2018;8(2):e020420.

11. Nye M, Sweeny A, Watkins S, Ingold J, Sharwood P. Difficult vascular access in hospitalised patients: delays to treatment, cannulation attempts, and use of ultrasound. Vascular Access (Osborne Park, WA Online). 2020;6(1):5-9.

12. Reigart JR, Chamberlain KH, Eldridge D, O'Brien ES, Freeland KD, Larsen $P$, et al. Peripheral intravenous access in pediatric inpatients. Clin Pediatr. 2012;51(5):468-72.

13. Kleidon TM, Cattanach P, Mihala G, Ullman AJ. Implementation of a paediatric peripheral intravenous catheter care bundle: A quality improvement initiative. J Paediatr Child Health. 2019;55(10):1214-23.

14. Witting MD. IV access difficulty: Incidence and delays in an urban emergency department. J Emerg Med. 2012;42(4):483-7.

15. Cooke M, Ullman AJ, Ray-Barruel G, Wallis M, Corley A, Rickard CM. Not "just" an intravenous line: Consumer perspectives on peripheral intravenous cannulation (PIVC). An international cross-sectional survey of 25 countries. PloS One. 2018;13(2):e0193436-e.

16. Asrar M, Al-Habaibeh A, Shakmak B, Shaw SJ. A device for improving the visual clarity and dimension of veins. Br J Nurs. 2018;27(19):S26-S36 doi: 10.12968/bjon.2018.27.19.S26.

17. Biswas J, , Drogin EY, Gutheil TG. Treatment delayed is treatment denied. J Am Acad Psychiatry Law. 2018;46(4):447-53.

18. Williams DO. Treatment delayed is treatment denied. Circulation. 2004;109(15):1806-8.

19. Sou V, McManus C, Mifflin N, Frost SA, Ale J, Alexandrou E. A clinical pathway for the management of difficult venous access. BMC Nurs. 2017;16(1):64.

20. Stolz LA, Stolz U, Howe C, Farrell IJ, Adhikari S. Ultrasound-guided peripheral venous access: a meta-analysis and systematic review. J Vasc Access. 2015;16(4):321-6.

21. Oliver G. Whose line is it anyway? Br J Nurs. 2015;24(Sup2):S3-S.

22. Denton A, Bodenham A, Conquest A, Davies A, Davidson A, Portsmouth $J$, et al. Standards for infusion therapy. 4th ed. London, UK: The Royal College of Nursing; 2016 [updated 2019]. Available from: http://eprints. hud.ac.uk/id/eprint/31627/

23. Brass P, Hellmich M, Kolodziej L, Schick G, Smith AF. Ultrasound guidance versus anatomical landmarks for internal jugular vein catheterization. Cochrane Database Syst Rev. 2015;2018(12):CD006962-CD.

24. Shiver S, Blaivas M, Lyon M. A prospective comparison of ultrasoundguided and blindly placed radial arterial catheters. Acad Emerg Med. 2006;13(12):1275-9.

25. McDicken WN, Anderson T. Basic physics of medical ultrasound. London: Churchill Livingstone; 2011. p. 3-15.

26. Troianos CA, Hartman GS, Glas KE, Skubas NJ, Eberhardt RT, Walker JD, et al. Guidelines for performing ultrasound guided vascular cannulation: recommendations of the American Society of Echocardiography and the Society of Cardiovascular Anesthesiologists. Anesth Analg. 2012;114(1):46-72

27. Blanco P. Ultrasound-guided vascular cannulation in critical care patients: a practical review. Med Intensiva. 2016;40(9):560-71.

28. Li X, Fang G, Yang D, Wang L, Zheng C, Ruan $L$, et al. Ultrasonic technology improves radial artery puncture and cannulation in intensive care unit (ICU) shock patients. Med Sci Monit. 2016;22:2409-16.

29. Gregg SC, Murthi SB, Sisley AC, Stein DM, Scalea TM. Ultrasound-guided peripheral intravenous access in the intensive care unit. J Crit Care 2010;25(3):514-9.

30. Archer-Jones A, Sweeny A, Schults JA, Rickard CM, Johnson L, Gunter A, et al. Evaluating an ultrasound-guided peripheral intravenous cannulation training program for emergency clinicians: an Australian perspective. Australas Emerg Care. 2020;23(3):151-6.

31. Soni NJ, Arntfield R, Kory P. Point of care ultrasound. 2nd ed. Philadelphia, PA: Elsevier; 2020.

32. Anantasit N, Cheeptinnakorntaworn P, Khositseth A, Lertbunrian R, Chantra M. Ultrasound versus traditional palpation to guide radial artery cannulation in critically ill children: a randomized trial. J Ultrasound Med. 2017;36(12):2495-501.

33. Benkhadra M, Collignon M, Fournel I, Oeuvrard C, Rollin P, Perrin M, et al. Ultrasound guidance allows faster peripheral IV cannulation in children under 3 years of age with difficult venous access: a prospective randomized study. Pediatr Anesth. 2012;22(5):449-54.

34. Bridey C, Thilly N, Lefevre T, Maire-Richard A, Morel M, Levy B, et al. Ultrasound-guided versus landmark approach for peripheral intravenous access by critical care nurses: a randomised controlled study. BMJ Open. 2018;8(6):e020220.

35. The Online Medical Device Exhibition [Catalogue]. 2020 [Hand-held ultrasound system]. Available from: www.medicalexpo.com/medicalmanufacturer/hand-held-ultrasound-system-17736.html.

36. Rykkje A, Carlsen JF, Nielsen MB. Hand-held ultrasound devices compared with high-end ultrasound systems: a systematic review. Diagnostics (Basel). 2019;9(2):61 10.3390/diagnostics9020061.

37. Kobayashi T, Kato H. Development of pocket-sized hand-held ultrasound devices enhancing people's abilities and need for education on them. J Gen Fam Med. 2016;17(4):276-88.

38. Medical Expo Vein Finder. Available from: https://www.medicalexpo. com/medical-manufacturer/infrared-vein-finder-55908.html.

39. Berings MG, Poell RF, Simons PR, van Veldhoven MJ. The development and validation of the on-the-job learning styles questionnaire for the nursing profession. J Adv Nurs. 2007;58(5):480-92.

40. Barth D, Nemec RM, Cho DD, Slomer A, Cojocari E, Kim K, et al. The practical integration of a hybrid model of ultrasound-guided peripheral venous access in a large apheresis center. J Clin Apher. 2020;35(4):32834.

41. ERCI Institute. Adoption of point-of-care ultrasound is outpacing safeguards. Hazard \#2-2020 top 10 health technology hazards. Health Devices. 2020.

42. Anderson S, Allen P, Peckham S, Goodwin N. Asking the right questions: scoping studies in the commissioning of research on the organisation and delivery of health services. Health Res Policy Syst. 2008;6(1):7.

43. Arksey H, O'Malley L. Scoping studies: towards a methodological framework. Int J Soc Res Methodol. 2005;8(1):19-32.

44. Peters MD, Godfrey CM, Khalil H, Mclnerney P, Parker D, Soares CB. Guidance for conducting systematic scoping reviews. Int J Evid Based Healthc. 2015;13(3):141-6.

45. Tricco AC, Lillie E, Zarin W, O'Brien K, Colquhoun H, Kastner M, et al. A scoping review on the conduct and reporting of scoping reviews. BMC Med Res Methodol. 2016;16(1):15-.

46. Damschroder LJ, Aron DC, Keith RE, Kirsh SR, Alexander JA, Lowery JC. Fostering implementation of health services research findings into practice: a consolidated framework for advancing implementation science. Implement Sci. 2009;4(1):50. 